\title{
Fenestration of Axillary Vein by a Variant Axillary Artery
}

\author{
Hadimani GA, Desai SD, Bagoji IB, Patil BS
}

\author{
Department of Anatomy \\ Sri BM Patil Medical College \\ BLDE University Bijapur \\ Karnataka, India
}

Corresponding Author

Gavishiddpaa A. Hadimani

Department of Anatomy

Sri BM Patil Medical College

BLDE University Bijapur

Karnataka, India

E-mail: gavish.hadimani@yahoo.com

Citation

Hadimani GA, Desai SD, Bagoji IB, Patil BS. Fenestration of Axillary Vein by a Variant Axillary Artery. Kathmandu Univ Med J 2013;42(2):162-164.

\begin{abstract}
Variations of venous pattern in the arm are common. In this case report, we present a variation of axillary artery and vein. During routine educational dissections of axillary region, it was observed that a fenestrated axillary vein was perforated by a variant axillary artery in right arm of an old male cadaver. The axillary artery which was fenestrated through axillary vein had only two branches arising from its second part and no branches from its remaining distal parts. The branches are thoracoacromial (usual) and another large collateral (unusual) branch. This collateral branch is the origin of several important arteries as the subscapular, circumflex scapular, posterior circumflex humeral and lateral thoracic arteries. We propose to name this artery as collateral axillary arterial trunk. The course of this collateral axillary arterial trunk and its branches and also clinical significance of this variation are discussed in the paper.
\end{abstract}

\section{KEY WORDS}

Axillary Artery, axillary vein, fenestration

\section{INTRODUCTION}

The axillary artery, a continuation of the subclavian artery, begins at the outer border of the first rib, and ends normally at the inferior border of teres major muscle where onwards it continues as the brachial artery. Pectoralis minor muscle crosses it and so divides it into three parts which are proximal, posterior and distal to the muscle. Conventionally, the proximal part (first part) gives superior thoracic artery, the posterior part (second part) gives thoraco-acromial and lateral thoracic arteries and distal part (third part) gives subscapular artery, anterior and posterior circumflex humeral arteries. ${ }^{1}$

The axillary vein lies along the medial side of the artery and is a continuation of the basilic vein. It begins at the inferior border of the teres major muscle and ends at the lateral border of the first rib, where it becomes the subclavian vein. It receives tributaries that parallel the branches of the axillary artery. The cephalic vein joins the axillary vein just before it becomes the subclavian vein.

There is an extensive collateral circulation associated with the subclavian and axillary arteries, particularly around the scapula. This clearly becomes of clinical significance during injury to the axillary artery. Here we present an unusual variation in which, a collateral branch arose from second part of the axillary artery and gave majority of the branches, which otherwise arise directly from the first and third parts of the axillary artery.

The axillary vein is an alternate route for venous access during pacemaker and cardioverter defibrillator (ICD) implantation, treatment of severe burns, and evaluation of central thoracic venous thrombosis caused by thoracic outlet compression, and treatment of breast carcinoma. ${ }^{2,3}$ Therefore, variations in this vein are of great clinical significance. 


\section{CASE REPORT}

During routine dissection for undergraduate students in the department of anatomy Sri B M Patil Medical College, Hospital and Research centre, BLDE University, Bijapur we found unilateral variations in right axillary artery and axillary vein. All the variations were dissected, examined and photographed. Variations include, Axillary artery gives only two branches from it. From the second part it gives one branch called thoracoacromial artery further it divides into pectoral, deltoid, acromial and clavicular arteries (fig $1)$.

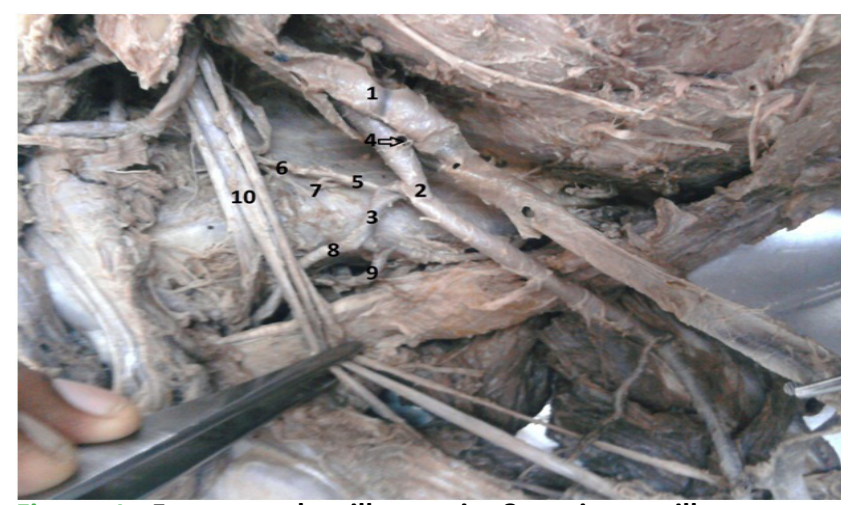

Figure 1. Fenestrated axillary vein \& variant axillary artery. 1-fenestrated axillary vein, 2-variant axillary artery with its only two branches, 3-collateral axillary artery, 4(aero)thoracoacromial artery (cut), 5-subscapular trunk, 6- subscapular artery, 7-superior thoracic artery, 8-posterior circumflex humeral artery, 9 -circumflex scapular artery, 10-brachial plexus.

From the second part of axillary artery a large collateral branch arose behind the pectoralis minor muscle, which gave many branches which otherwise arise from the second and third parts of the axillary artery and we propose to name this artery as Collateral Axillary Artery.

The Collateral Axillary Arterial trunk descends medial to the axillary vein and medial root of the median nerve, resting on ventral surface of subscapular muscle under cover of pectoralis minor muscle. At the distal border of the Subscapularis muscle, this collateral trunk ends by bifurcating into two branches.

The Collateral Axillary Arterial trunk gave common subscapular trunk which later divides into subscapular artery supplies subscapular muscle and superior thoracic artery.

Posterior circumflex humeral artery arose from collateral axillary arterial trunk after common subscapular trunk and passes through quadrangular space.

Circumflex scapular artery arises from collateral axillary arterial trunk (normally it is branch from subscapular artery) runs behind the scapula after passing through superior triangular space.

Finally collateral axillary arterial trunk bifurcating into two branches one continues as lateral thoracic artery runs in front of subscapularis muscle and other branch supplies subscapularis muscle.

Before branching, axillary artery passes through the fenestra formed by the axillary vein. Basilic vein continues as axillary vein. Brachial veins draining into axillary vein, this axillary vein is bifurcating and rejoining and forming fenestra through which axillary artery is passing. Fenestra of axillary vein is lying behind the pectoralis minor muscle (fig 2). All the tributaries of axillary vein which drains axillary region are draining into fenestrated axillary vein.

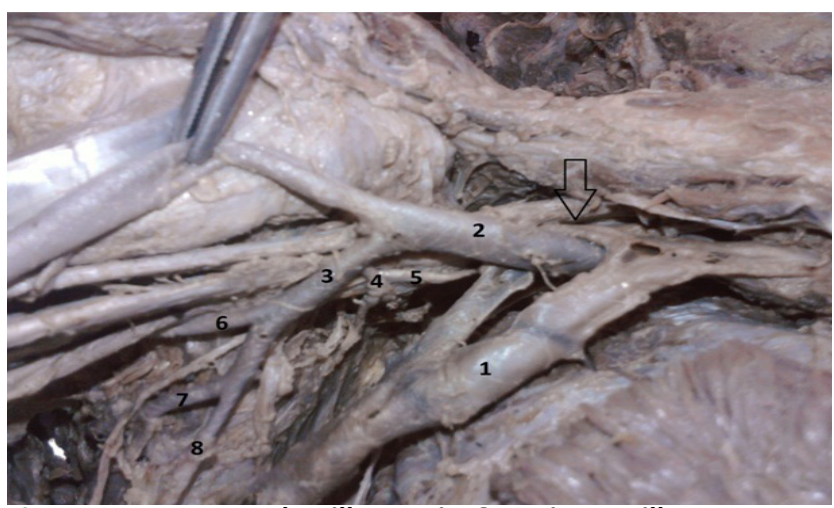

Figure 2. Fenestrated axillary vein \& variant axillary artery. 1 -fenestrated axillary vein, 2-variant axillary artery 3-collateral axillary artery, 4 -common subscapular trunk, 5 -superior thoracic artery, 6 -posterior circumflex humeral artery, 7 circumflex scapular artery, 8 -lateral thoracic artery. Aero indicates fenestration of axillary vein.

\section{DISCUSSION}

Anatomic variations in the major vessels of the upper limb have been reported earlier. It is not uncommon to find the variation in the branching pattern of axillary vessels. The review of literature shows many variations, in which two or more branches arising from the common trunk are reported. However, variant axillary artery which is passing through fenestra formed by axillary vein has not been reported to the best of our knowledge.

A case report by Vasuda S. et al. reported presence of two branches from axillary artery one is superior thoracic artery other is common subscapular trunk. Common subscapular trunk gives all the remaining branches of axillary artery. ${ }^{4}$ A case reported by Venieratos and Lolis shows common subscapular trunk gave origin to circumflex scapular, thoracodorsal, anterior and posterior circumflex humeral, profunda brachii and ulnar collateral arteries. ${ }^{5}$ In another report by Samuel et al.the third part of the axillary artery gave a common arterial trunk, which further gave anterior and posterior circumflex humeral, subscapular, radial collateral, middle collateral and superior ulnar collateral arteries with absence of profunda brachii artery. Our present report differs from this earlier report in branching pattern. ${ }^{6}$

According to personal experience of Prades et al. the clinical incidence of the fenestration anomaly is approximately $0.4 \%$ of unilateral neck dissections. They reported three 
intra-operative cases in which the lateral branch of the accessory nerve (XI) always passed medially to the anterior vein and laterally to the posterior vein, between the venous duplication. ${ }^{7}$

Tanju et al. conducted a study in order to investigate the diagnostic value of direct contrast-enhanced 3D MR venography in mapping the upper extremity venous system and planning invasive radiological and surgical procedures. They encountered venous pathology in 16 of the 19 cases (84\%). Furthermore they observed two cases of duplication of cephalic vein and one case of internal jugular vein fenestration, being totally three (15\%) cases of anatomic venous variations. ${ }^{8}$ Fenestration of various vessels are observed in many studies. ${ }^{9,10}$ However we did not find fenestrations of axillary vein by a variant axillary artery in literature.

In the embryologic term, a dense capillary plexus forms initially in the limb bud mesenchyme. As a result of different hemodynamic influences, some anastomosis develop between superficial and deep vessels, where as others do not develop or regress. In this way, the superficial and deep

\section{REFERENCES}

1. Standring, S, Johnson D, Ellis H, Collins P. Gray's Anatomy. 39th Ed. London: Churchill Livingstone; 2005. p.856.

2. Decker GAG, du Plessis DJ. Lee McGregor's. Synopsis of Surgical Anatomy. 12th ed. Bristol: John Wright \& Sons Ltd; 1986.

3. Moore KL, Dalley AF. Clinically oriented Anatomy. 4th ed. Philadelphia: Lippincott Williams \& Wilkins; 1999.

4. Vasudha S, Theresa J, Sampath M, Rajanigandha V. Shruti S. Abnormal Branching of the Axillary Artery: Subscapular Common Trunk. A Case Report Int. J. Morphol 2008;26(4):963-966.

5. Venieratos, D, Lolis, E. D. Abnormal ramification of the axillary artery: sub-scapular common trunk. Morphologie 2001;85(270):23-4.

6. Samuel V. P, Vollala V. R, Nayak S, Rao M, Bolla S.R, Pammidi N. A rare variation in the branching pattern of the axillary artery. Indian J. Plast. Surg 2006; 39:222-3.

7. Prades JM, Timoshenko A, Dumollard JM, Durand M, Merzougui $\mathrm{N}$, Martin C. High duplication of the internal jugular vein: clinical incidence in the adult and surgical consequences, a report of three clinical cases. SurgRadiolAnat 2002; 24: 129-32. blood vessels arise. The superficial capillary plexus persists for the development of superficial veins of the upper limb. The seventh cervical segmental artery gives rise to axillary artery and any abnormality during development results in the unusual branching pattern. ${ }^{11}$ Therefore, there is the possibility that axillary artery will become entrapped within the venous plexus to give rise to the variant morphology in the present case.

Veins of the upper limb are commonly used as an intravenous route for total parenteral nutrition, fluid and electrolyte infusion, therapeutic invasive procedures, blood samples, blood transfusion, catheterization and occasionally for grafting procedures. A large number of invasive procedures, both diagnostic and therapeutic, are carried out using veins of the upper limb, particularly in and distal to the axillary region. ${ }^{12}$ The axillary vein is an alternate route for venous access during pacemaker and cardioverter defibrillator (ICD) implantation, treatment of severe burns, evaluation of central thoracic venous thrombosis caused by thoracic outlet compression, and treatment of breast carcinoma. ${ }^{2,3}$
8. Tanju S, Sancak T, Düflünceli E, Yaømurlu B, Erden I, SanlidilekU. Direct contrast-enhanced 3D MR venography evaluation ofupper extremity deep venous system. Diagn Interv Radiol 2006; 12:74-9.

9. Gardiner KJ, Irvine BW, Murray A. Anomalous relationship of the spinal accessory nerve to the internal jugular vein. ClinAnat 2002;15: 62-3.

10. Alaani A, Webster K, Pracy JP. Duplication of internal jugular veinand relation to the spinal accessory nerve. Br J Oral MaxillofacSurg 2005; 43: 528-31.

11. Wollard, H. H. The development of the principal arterial stems in the forelimb of the pig. Contrib. Embryol 1922;14: 139.

12. Andel H, Rab M, Felfernig M. The axillary vein central venous catheter in severely burned patients. Burns 1999; 25: 753-6. 